\title{
Measuring the Effects of Stress on Mobile Interaction
}

\author{
ZHANNA SARSENBAYEVA, The University of Melbourne, Australia \\ NIELS VAN BERKEL, The University of Melbourne, Australia \\ DANULA HETTIACHCHI, The University of Melbourne, Australia \\ WEIWEI JIANG, The University of Tokyo, Japan \\ TILMAN DINGLER, The University of Melbourne, Australia \\ EDUARDO VELLOSO, The University of Melbourne, Australia \\ VASSILIS KOSTAKOS, The University of Melbourne, Australia \\ JORGE GONCALVES, The University of Melbourne, Australia
}

Research shows that environmental factors such as ambient noise and cold ambience can render users situationally impaired, adversely affecting interaction with mobile devices. However, an internal factor which is known to negatively impact cognitive abilities - stress - has not been systematically investigated in terms of its impact on mobile interaction. In this paper, we report a study where we use the Trier Social Stress Test to induce stress on participants, and investigate its effect on three aspects of mobile interaction: target acquisition, visual search, and text entry. We find that stress reduces completion time and accuracy during target acquisition tasks, as well as completion time during visual search tasks. Finally, we are able to directly contrast the magnitude of these effects to previously published effects of environmentally-caused impairments. Our work contributes to the growing body of literature on situational impairments.

CCS Concepts: • Human-centered computing $\rightarrow$ Empirical studies in HCI; • Human-centered computing $\rightarrow$ Ubiquitous and mobile computing; Smartphones.

Additional Key Words and Phrases: Smartphones, situational impairments, mobile interaction, stress, performance, Trier Social Stress Test

ACM Reference Format:

Zhanna Sarsenbayeva, Niels van Berkel, Danula Hettiachchi, Weiwei Jiang, Tilman Dingler, Eduardo Velloso, Vassilis Kostakos, and Jorge Goncalves. 2019. Measuring the Effects of Stress on Mobile Interaction. Proc. ACM Interact. Mob. Wearable Ubiquitous Technol. 3, 1, Article 24 (March 2019), 18 pages. https://doi.org/10.1145/3314411

Authors' addresses: Zhanna Sarsenbayeva, The University of Melbourne, Melbourne, Australia, z.sarsenbayeva@student.unimelb.edu.au; Niels van Berkel, The University of Melbourne, Melbourne, Australia, n.vanberkel@student.unimelb.edu.au; Danula Hettiachchi, The University of Melbourne, Melbourne, Australia, dhettiachchi@student.unimelb.edu.au; Weiwei Jiang, The University of Tokyo, Tokyo, Japan, wjiang@akg.t.u-tokyo.ac.jp; Tilman Dingler, The University of Melbourne, Melbourne, Australia, tilman.dingler@unimelb.edu.au; Eduardo Velloso, The University of Melbourne, Melbourne, Australia, eduardo.velloso@unimelb.edu.au; Vassilis Kostakos, The University of Melbourne, Melbourne, Australia, vassilis.kostakos@unimelb.edu.au; Jorge Goncalves, The University of Melbourne, Melbourne, Australia, jorge.goncalves@unimelb.edu.au.

Permission to make digital or hard copies of all or part of this work for personal or classroom use is granted without fee provided that copies are not made or distributed for profit or commercial advantage and that copies bear this notice and the full citation on the first page. Copyrights for components of this work owned by others than the author(s) must be honored. Abstracting with credit is permitted. To copy otherwise, or republish, to post on servers or to redistribute to lists, requires prior specific permission and/or a fee. Request permissions from permissions@acm.org.

(c) 2019 Copyright held by the owner/author(s). Publication rights licensed to ACM.

2474-9567/2019/3-ART24 \$15.00

https://doi.org/10.1145/3314411

Proc. ACM Interact. Mob. Wearable Ubiquitous Technol., Vol. 3, No. 1, Article 24. Publication date: March 2019. 


\section{INTRODUCTION}

Due to the ubiquitous nature of smartphones, users interact with them under varying environmental (e.g., in noisy environments) and internal (e.g., under stress, in different moods) conditions. Previous research has shown that environmental and contextual factors, such as cold ambience [12], ambient noise [44], encumbrance [38], and walking [11] negatively affect mobile interaction and lead to situational impairments. This has given rise to a growing body of work in HCI that aims to enable smartphones to detect when the user is situationally impaired and subsequently adapt the interface to mitigate the effects of such impairments during mobile interaction [43, 46, 57].

Although prior research has emphasised the importance of understanding the effects of situational impairments $[43,49,63]$, these studies mostly focus on environmental factors, such as cold ambience. In contrast, relatively little work has investigated the effect of an important internal factor on mobile interaction: stress, a mental state often experienced on a daily basis [1], which has been shown to negatively impact performance in everyday activities (e.g., $[16,22,39])$.

In this paper, we investigate the effect of stress on task performance in smartphones. According to Lazarus et al. [23], stress can be defined in terms of the relationship between an individual and an environment or situation [51]. Given this notion [23] and the temporal effect of certain stressors (personal or work-related) on human behaviour [26,55], stress has been identified as a potential cause of situational impairment that is likely to have an impact during mobile interaction [43].

In our study, we use the Trier Social Stress Test (TSST) [20] to induce stress in our participants in order to measure its effect on three mobile interaction tasks: target acquisition, visual search, and text entry [44]. We show that stress significantly reduces target access time and accuracy during target acquisition tasks compared to the baseline, as well as completion time during visual search tasks. Further, we directly contrast the magnitude of stress-induced situational impairments to other situational impairments based on results in the literature, namely cold ambience [42] and ambient noise [44].

The main contributions of this paper are to quantify the effect of stress on mobile task performance, compare its effect size to that of cold- and noise-induced situational impairments, and, hence, to contribute to the growing body of research on situational impairments.

\section{RELATED WORK}

Previous work has highlighted that different environmental and contextual factors, such as cold ambience [12], ambient noise [44], mobile state of the user [11], and encumbrance [38] can negatively affect mobile interaction. These factors can cause users to experience situational impairments and, thereby, impact their performance during mobile interaction [43]. For example, cold ambient temperature has been shown to adversely affect smartphone input performance [12, 42]. In particular, throughput and accuracy drop significantly when performing acquisition tasks under cold temperatures. Other studies have shown a negative effect of walking on text entry $[11,35]$ and target acquisition tasks [48]. User encumbrance also negatively affects mobile interaction, resulting in decreased accuracy, longer target acquisition times, and increased error rate [38].

Sarsenbayeva et al. [44] measured the effect of background noise on smartphone interaction performance. The types of background noise presented in that study included music with fast and slow tempo, urban indoor and outdoor noise, and meaningful and meaningless speech. Similar to our study, mobile input performance was measured in terms of three common smartphone activities: target acquisition, visual search, and text entry. Performance under ambient noise conditions was benchmarked against the silent condition. The authors found that music reduced completion time during target acquisition tasks, while urban noise and speech increased the text entry rate. Although a number of previous studies have investigated the effect of stress on interaction with stationary technology (e.g., keyboard and mouse), the effect of stress on mobile interaction remains underexplored. For example, Karunaratne et al. [18] reported stronger keyboard taps amongst participants exposed to 
stressful conditions. Furthermore, Rodrigues et al. [40] investigated the effect of stress on mouse movements. The authors found that stressed students, when presented with challenging questions, carried out significantly more mouse movements [40].

In our study, we contribute to the growing body of research on situational impairments [43] by quantifying the effect of stress on interaction with smartphones, an internal factor that has been shown to adversely impact performance when using other technologies, such as desktop computers [18, 40,62]. We achieve this by using the TSST protocol [20] to induce stress in our participants, and measure changes to their performance while completing typical smartphone tasks.

\subsection{Cognitive and Physiological Effects of Stress}

Behavioural and psychological scientists study the effect of stress on humans' daily lives for a variety of reasons. For example, researchers have aimed to identify the events leading to stress and how negative outcomes of cumulative stress can be avoided [14].

Early work, showed a curvilinear relationship between arousal and task performance, particularly with very high and very low levels of arousal inhibiting task performance, whereas moderate levels facilitate it [65]. More recently, research has shown the negative effect of stress on human cognitive performance [67]. For example, stress negatively affects working memory [27, 67] and verbal declarative memory [21, 37]. In addition, studies by Payne et al. [39] and Jelici et al. [16] report adverse effects of stress on human cognition resulting in memory impairments. Lupien et al. [26] also show that under stressful conditions, declarative memory is significantly impaired compared to a non-stressful condition in a word-pairs recall task. Moreover, Kuhlmann et al. [22] show that acute stress significantly delays memory retrieval. They utilise the TSST, a widely used protocol for inducing stress in participants through two tasks: public speaking and arithmetic subtraction [20]. They then asked their participants to recall pairs of words memorised at the beginning of the experiment. The results of this study show that stressed participants have significantly reduced memory retrieval as compared to the control condition [22]. Wolkowitz et al. [64] report similar results showing that stress has a detrimental effect when recalling words from a previously learnt list.

Further, Marquart [29] observed that stress decreased participants' learning ability and results in non-adaptive behaviour (e.g., longer reaction time when completing cognitive tasks [23]). Verville et al. [61] found that participants who were exposed to a stressful condition required significantly longer time for recognising pictures that were flashed on a screen as compared to a control group. This, however, is a contested finding as other studies have shown that stress sped up participants' performance during an image sorting task (e.g., [33]). In addition, a reduction of task completion time resulted in a greater number of errors [33]. These results are similar to a study by McKinney [32], where stress increased the number of errors in completing multiplication problems and a learning syllables task.

Based on this rich literature we hypothesise that stress will hinder user performance in smartphone tasks. In particular, participants under stress will be less accurate when compared to a baseline measurement.

\subsection{Stress Detection Methods}

Stress induces biological responses, which can thus be physiologically measured. Examples include changes in skin conductivity [50], heart rate variability [56], muscle tension [56], and heart rate [47]. Several studies have focused on the development of wearable technology that allows for the unobtrusive tracking of physiological measures. As a result, a variety of wearables exists that track physiological measures (e.g., Empatica E4 wristband ${ }^{1}$, Oura ring ${ }^{2}$.

\footnotetext{
${ }^{1}$ https://www.empatica.com/research/e4/

${ }^{2}$ https://ouraring.com/
} 
Several studies have attempted to detect stress from people's interaction behaviour using sensor-based technologies [3, 14, 41]. For example, Hernandez et al. use a pressure-sensitive keyboard and a capacitive mouse to detect the effect of stress during computer interaction. They found that more than half of their participants increased their typing pressure under the stressful condition when compared to the control condition. They also showed that the majority of participants (75\%) covered more of the surface of the mouse when they were stressed [14]. Furthermore, Exposito et al. [10] suggest using iPhone's built-in keyboard pressure sensor to detect stress. The authors show that under stressed conditions, participants typing pressure was higher when compared to non-stressed conditions [10]. Prior research has also shown that the combination of wearable sensors and smartphone interaction patterns can detect stress more accurately. For instance, Sano and Picard [41] achieved a higher accuracy rate for stress detection (75\%) compared to previous results $(53 \%)$ that only used smartphone interaction patterns [3]. Other stress detection methods are based on self-reports and questionnaires. For example, Cohen et al. [9] suggest the use of perceived stress as an objective stress measure. Further, Spielberger and colleagues recommend asking participants to self-report their anxiety levels using the State-Trait Anxiety Inventory (STAI) to identify the occurrence of stress [54].

In our study, we collect both sensor and self-report data to validate that we are indeed inducing stress in our participants throughout the experiment. Namely, we use heart rate variability (HRV) derived from the data of an Empatica E4 and collect self-report data using the STAI questionnaire.

\section{METHOD}

In this study, we investigate the effect of stress on performance on three common mobile interaction tasks: target acquisition, visual search, and text entry. We use the experimental tasks developed in the study by Sarsenbayeva et al. [44]. This allows us to directly compare the effects of stress-induced situational impairments with those previously reported for cold-induced and noise-induced situational impairments. We describe each task in detail below.

\subsection{Smartphone Tasks}

We used a Samsung Galaxy S7 smartphone (Android 7.0) with a 5.1-inch screen $(1080 \times 1920 \mathrm{px})$ in this experiment. This smartphone model was chosen in order to have an identical screen size and screen resolution to the smartphone used in previous studies by Sarsenbayeva et al. [42, 44] on situational impairments. The three tasks were presented to participants in a random order to avoid sequence effects. Participants first completed extensive training until they were comfortable with each of the presented tasks, thus minimising any potential learning effects. Both the training and actual tasks were completed while standing, and participants were instructed to use only the index finger of their dominant hand for interaction, while holding the smartphone with their non-dominant hand.

3.1.1 Target Acquisition Task. In the target acquisition task participants are asked to tap circular targets (radius $=135 \mathrm{px}$ ) which appear one at a time at random locations on the screen. Each circle has an indicated centre and participants are asked to tap the indicated center of the targets as precisely and quickly as possible. We log the coordinates of the target's centre, the participant's touch point, and the elapsed time. The interface of the task is shown in Figure 1-A.

3.1.2 Visual Search Task. In the visual search task, participants must find a target icon amongst 24 other icons $(100 \times 100 \mathrm{px})$, arranged according to a $4 \times 6$ grid [13]. The target icon is first shown, participants can then take as much time as needed to memorise it (Figure 1-B). In the subsequent screen they must find and tap the memorised target among 24 icons (Figure 1-C). To minimise any potential learning effects, the application ensures that the target is randomly selected and remaining icons are randomly distributed across the grid. 


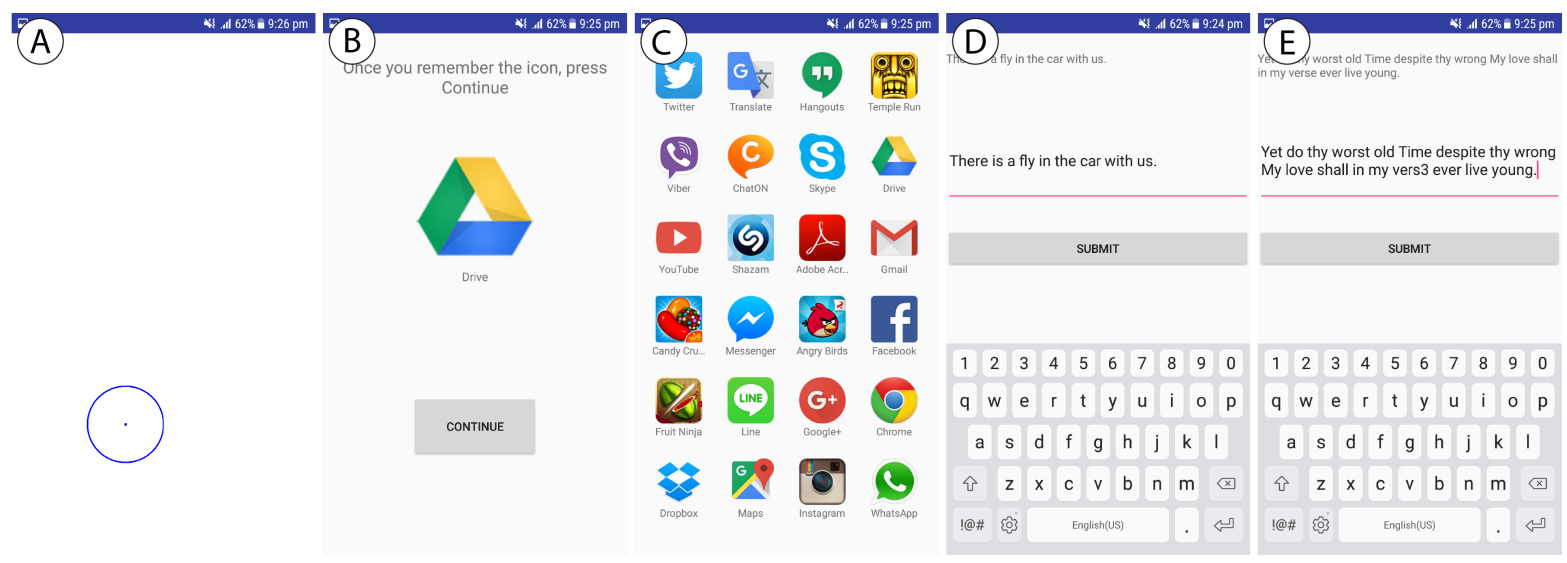

Fig. 1. Interface of the application with Target Acquisition Task (A), Visual Search Task (B-C), and Text Entry Task with user's input for easy and difficult texts (D-E).

3.1.3 Text Entry Task. In the text entry task, our participants are presented with a snippet of text (displayed on the top-half of the screen), which they must re-type in a text box. The texts are of varying difficulty: 1) easy consisting of only one sentence with common words widely used on a daily basis (see Figure 1-D), 2) difficult - consisting of several sentences chosen from Shakespeare's sonnets (see Figure 1-E). In total we have 10 easy sentences and 10 difficult sentences, and at each round of tasks a sentence is randomly selected by the application. We validated the difficulty of the sentences with the Flesch-Kincaid readability test [19]. Easy sentences had an average grade of 1.6 and difficult sentences had an average grade of 5.1.

\subsection{Participants}

We recruited 24 participants between 20 and $55(M=31, S D=9.5)$ years old through our university's mailing lists and snowball recruitment with equal number of male and female participants to avoid gender bias [59]. Participants had a diverse range of educational backgrounds (e.g., Electrical, Mechanical, Infrastructure and Civil Engineering, Agriculture, Entomology, Architecture, and Computer Science).

\subsection{Procedure}

To induce stress in our participants, we followed the TSST protocol [20]. TSST is a validated protocol used to induce stress in study participants that has been widely used in Psychology research. The original TSST protocol [20] describes three main stress points in the experiment: following a relaxation period (20 minutes), following stress-inducing tasks (speech and arithmetic), and following post-stress recovery. Hence, we ask participants to complete the smartphone tasks at these three points. The overview of the experimental procedure is presented in Figure 2.

In order to validate whether our participants experienced stress throughout the experiment, we calculated their heart rate variability (HRV) from the Empatica E4 wristband data, as HRV has been shown to correlate with a person's stress levels [56]. We also collected self-reported anxiety levels of the participants via the STAI questionnaire [54], a tool commonly used by researchers conducting studies with the TSST to validate fluctuations in stress levels. Sensor data was then cross-referenced with smartphone data using timestamps.

In line with the TSST protocol, participants were not aware that the study investigated the effect of stress on mobile interaction, and were not informed that we would induce stress on them to avoid triggering anxiety-related 


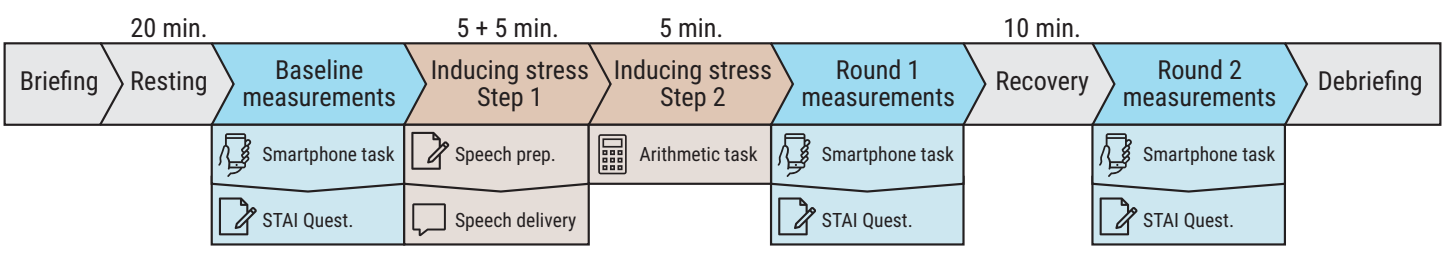

Fig. 2. Experimental Procedure.

behaviours. Again, according to the TSST protocol, this is done to reduce participant bias and to let us observe the natural reaction of participants to stress. The experimental design was approved by the Ethics Committee of our university. The experiment duration was approximately 90 minutes per participant, including briefing, training, data collection, and final interview. Each participant received a $\$ 30$ gift voucher for participation.

3.3.1 Participant Briefing. The experiment took place at our institution's usability lab, with an experimental setup consisting of two adjacent rooms, as recommended by the TSST protocol. One room works as a desensitisation room, where participants can rest and relax, while the other room is used for the stress-inducing tasks. Upon arrival, we welcomed our participants to the desensitisation room. We then informed them that we are investigating their cognitive performance as measured by their presentation skills and arithmetic skills, as well as their performance in a range of smartphone tasks. We then asked participants to sign a consent form agreeing to participate in the study and collected their demographic data (age, gender, background, dominant hand). Next, we instrumented our participants with an Empatica E4 wristband, and instructed them to wear it throughout the experiment on their non-dominant hand. Following this setup we asked our participants to complete all smartphone tasks for training purposes. Participants trained with all three study tasks in random order until they were comfortable with each one.

3.3.2 Baseline Measurements. After training, participants were left alone for 20 minutes, so that they could rest and stabilise. During this period we provided them with reading material with neutral content to keep them occupied and not cause any anxiety (descriptions and photographs of plants growing in different regions of the world), as recommended in the TSST protocol [20]. We also asked them not to use their smartphone during this time. Following, the participants were asked to complete a round of tasks on a smartphone. The purpose of this stage was to create a baseline measurement of performance while not stressed. After finishing the tasks, the participants completed the STAI questionnaire [54] to measure their perceived anxiety state.

3.3.3 Inducing Stress - Step 1. We guided our participants to the experiment room. Here, we provided them with a scenario according to which they were applying for their dream job and needed to attend a hiring interview. Participants were given 5 minutes to prepare a speech to convince a panel as to why they were the best candidate for their dream job. We also informed them that their performance was going to be video-recorded and reviewed by judges trained in public speaking. Once the preparation time was over, the participants were asked to deliver their speech in front of the panel of three judges, who were, unbeknownst to the participants, confederates of the research team. Participants had to speak for the entire 5 minutes, during which the panel members did not give any feedback and maintained neutral facial expressions.

3.3.4 Inducing Stress - Step 2. Upon completing step 1, participants were asked to perform an arithmetic task. We asked them to subsequently decrement 1022 by 13 and to say the number sequence out loud. The task is inspired by the 'Serial Sevens' tasks [52], which is used in clinical tests to assess mental functions, and lasts for 5 minutes. In case of miscalculation, a researcher informed the participants of their error and they were asked to restart the task from 1022. The purpose of the arithmetic task was to induce further stress on participants. 
After completing the arithmetic task, the participants completed a round of tasks on a smartphone under stress, followed by the STAI questionnaire (Round 1).

3.3.5 Post-Stress Recovery. At this stage, we brought participants back to the desensitisation room and left them alone for 10 minutes to rest and recover. We then asked them to complete a final round of smartphone tasks and complete the STAI questionnaire (Round 2). According to the TSST protocol [20], cortisol level reaches its peak during this moment of the experiment and, therefore, we wanted to assess their performance during mobile interaction at this point.

3.3.6 Participant Debriefing. Finally, we debriefed participants about the real purpose of the study and explained that it was expected to experience stress during the experiment. Further, we clarified that the tasks performed during the experiment were unreasonably difficult and did not reflect upon their aptitude or ability. We also informed them that we did not video-record their performance. We then conducted a short, semi-structured interview with our participants, asking them to report on their subjective perception of the experiment and the effect of stress on their performance.

\section{RESULTS}

In this section, we report our results regarding the effect of stress on our participants' performance across target acquisition, visual search, and text entry tasks. We also report participants' subjective assessments of their performance in the aforementioned smartphone tasks.

\subsection{Validation of Stress Occurrence}

We used HRV to validate the presence of stress in our participants and followed the process, described by McDuff and colleagues [31]. To generate HRV data from the bio-signals collected with the Empatica E4 sensor, we performed the generic HRV analysis method as follows. First, we interpolated and re-sampled inter-beat interval (IBI) data at $4 \mathrm{~Hz}$, to align the signals in a uniform time interval. Then we obtained an HRV power spectrum in time series from the detrended interpolated IBI data, by applying the Fast Fourier Transform (FFT) algorithm with a 512-sized slide window (i.e., a 512-sample or 128-second segment of a signal). We calculated the $\mathrm{HF}$ (High-Frequency) powers of the HRV as the summation of the discrete points corresponding to the power spectrum under $0.15-0.40 \mathrm{~Hz}$. As HF HRV was previously shown to be a reliable indicator of stress occurrence [4], we used it for further validation of presence of stress in our participants. We acknowledge that HRV can be a result of multiple factors apart from stress; however, we argue that in combination with the established stress-inducing protocol and participants' self-reports, we were able to reliably validate the occurrence of stress during our experiment. In addition, we did not collect EDA data due to the following reasons. First, EDA data collected from the wrist is shown to describe thermoregulatory relevant electrodermal phenomena, rather than the psychophysiological nature of the response [8]. Second, EDA is better suited for validating stress caused by discrete events (e.g., electrical shock) $[7,8,51]$, whereas in our study participants experienced longitudinal cumulative stress.

We applied a one-way repeated measures ANOVA, which yielded a statistically significant effect of stress on heart rate variability $(F(2,24)=6.54, p<0.01)$. A Tukey HSD post-hoc comparison test (with Bonferroni corrections) reveals that HRV values during Stress Induction $(M=32.84, S D=20.89)$ and Post-stress Recovery periods $(M=37.24, S D=18.69)$ are significantly lower $(p<0.01)$ compared to HRV values during the baseline measurements $(M=40.81, S D=26.05)$. Since low values of HF HRV indicate a physiological presence of stress [15], we can conclude from the HRV results that the participants were stressed after undergoing both the speech and arithmetic subtraction tasks of the protocol. The mean values for HF HRV are visualised in Figure 3. 


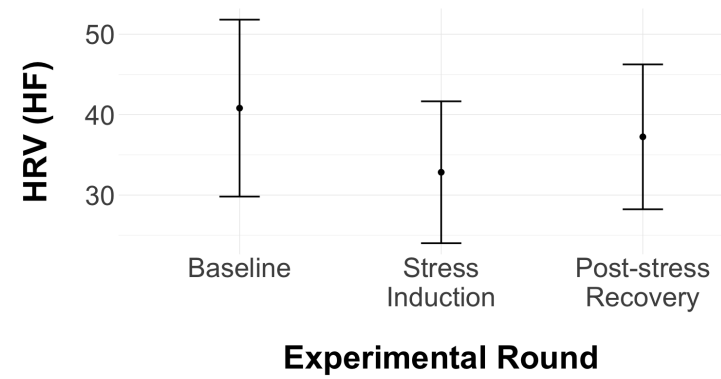

Fig. 3. HRV (HF) Mean Values (95\% Confidence Interval (CI))

Next, we examined participants' self-reported anxiety values as measured by the STAI questionnaire as collected following completion of each round of smartphone tasks. A one-way repeated measures ANOVA yielded a statistically significant effect of stress on self-reported anxiety values $(F(2,24)=10.23, p<0.01)$. Post-hoc comparisons using the Tukey HSD test (with Bonferroni corrections) indicated that participants were significantly more anxious $(p<0.01)$ during Stress Induction period $(M=37.08, S D=11.77)$ of the experiment compared to the baseline values $(M=30.71, S D=6.37)$. Furthermore, participants reported feeling significantly less anxious $(p<0.01)$ during Post-stress Recovery period $(M=31.79, S D=8.64)$ when compared to Stress Induction period. The mean values for self-reported anxiety levels are presented in Figure 4.
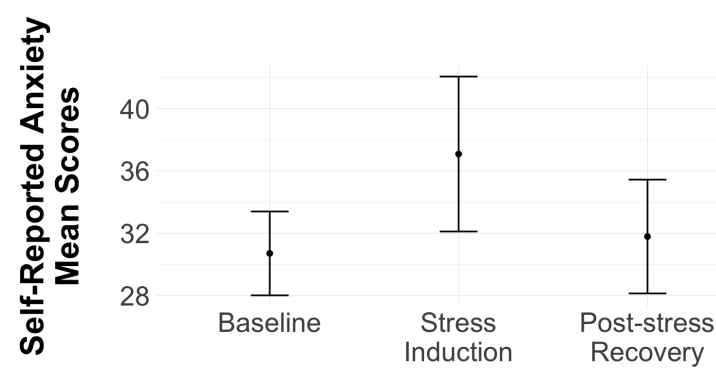

\section{Experimental Round}

Fig. 4. Self-Reported Anxiety Mean Values (95\% Cl)

From the sensor data and self-reported anxiety values we can conclude that the study protocol performed as intended in terms of inducing stress in participants.

\subsection{Target Acquisition Task}

We measured the target acquisition performance in terms of target acquisition time (ms), offset (px) between the target centre and the touch point, and effective throughput ${ }^{3}$. First, we applied a one-way repeated measures ANOVA to investigate the effect of stress on target acquisition time. The result showed a statistically significant

\footnotetext{
${ }^{3} \mathrm{~A}$ measure of human performance in completing target selection tasks that describes the relationship between the difficulty of the task and target acquisition time [28]
} 
effect of stress on time taken to hit a target $(F(2,24)=17.05, p<0.01)$. Post-hoc comparisons using the Tukey HSD test (with Bonferroni corrections) indicate that participants take significantly less time to tap a circle in Stress Induction $(M=534.77, S D=124.43, p<0.01)$ and Post-stress recovery periods $(M=520.18, S D=126.52, p<$ $0.01)$ as compared to the baseline $(M=538.68, S D=127.58)$. However, we found no statistically significant difference between time taken to hit a circle between Stress Induction and Post-stress Recovery periods. Mean target acquisition time values are presented in Figure 5.

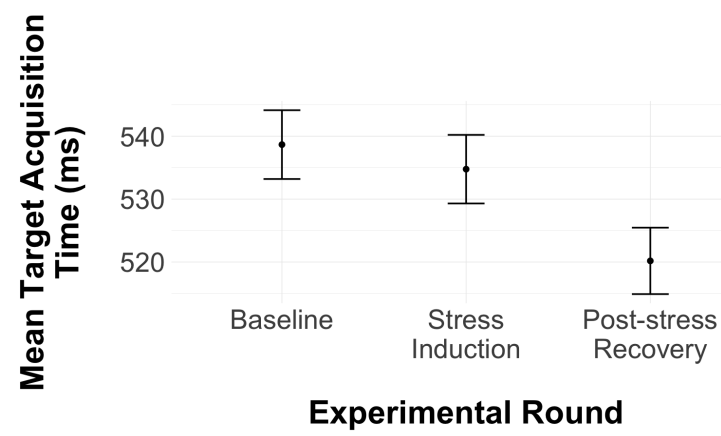

Fig. 5. Mean Target Acquisition Time (95\% Cl)

Then, we investigated the effect of stress on the touch accuracy during target acquisition. A one-way repeated measures ANOVA showed a statistically significant effect of stress on the offset size $(F(2,24)=20.11, p<0.01)$. Post-hoc comparisons using the Tukey HSD test (with Bonferroni corrections) indicate that touch offset size is significantly larger in Stress Induction $(M=49.14, S D=25.94, p<0.01)$ and Post-stress Recovery periods $(M=50.20, S D=26.51, p<0.01)$ as compared to the Baseline value of the offset size $(M=46.47, S D=25.57)$. However, we found no statistically significant difference in offset size between Stress induction and Post-stress Recovery measurements $(p>0.05)$. Mean offset size values are visualised in Figure 6.

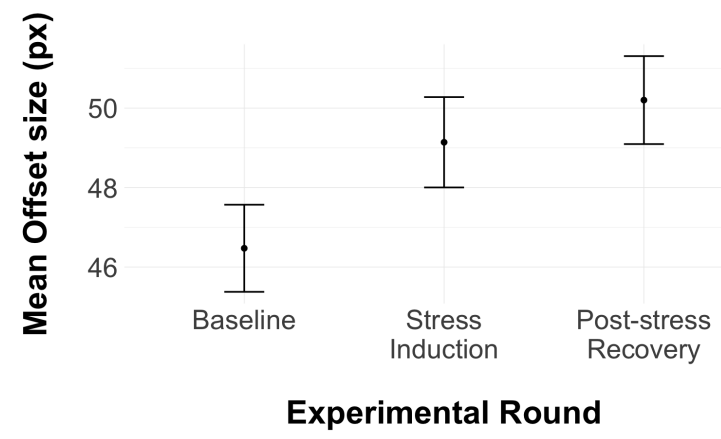

Fig. 6. Mean Offset Size $(95 \% \mathrm{Cl})$

Finally, we studied the effect of stress on effective throughput. Effective throughput was calculated as suggested by Soukoreff and MacKenzie [53]. A one-way repeated measures ANOVA did not show a statistically significant effect of stress on the effective throughput during target acquisition tasks $(p>0.05)$. 


\subsection{Visual Search Task}

We measured the performance during the visual search task in terms of the time taken to memorise the target icon, and the time taken to find the target icon. A one-way repeated measures ANOVA showed a statistically significant effect of stress on the time taken to memorise an icon $(F(2,24)=31.77, p<0.01)$. Post-hoc comparisons using the Tukey HSD test (with Bonferroni corrections) indicated that participants take less time to memorise an icon in Stress Induction $(M=714.52, S D=229.27, p<0.01)$ and Post-stress Recovery periods $(M=702.31, S D=$ $200.85, p<0.01)$ when compared to the baseline measurement $(M=800.51, S D=331.15)$. However, we found no statistically significant difference in the time taken to memorise an icon between the values measured during Stress Induction and Post-stress Recovery $(p>0.05)$. Mean values for time taken to memorise an icon are visualised in Figure 7.

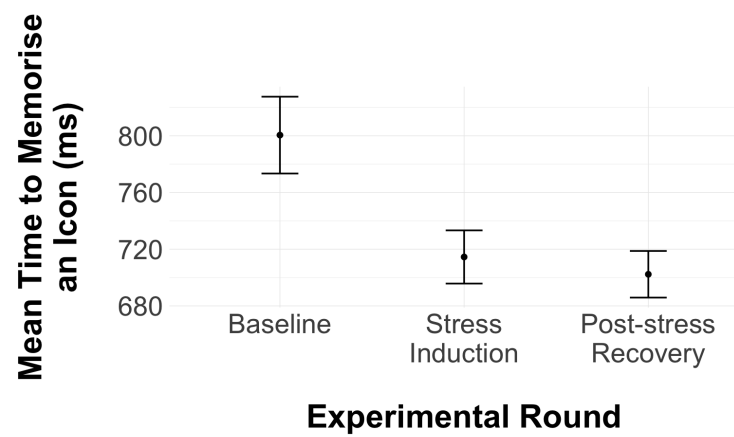

Fig. 7. Mean Time to Memorise an Icon $(95 \% \mathrm{Cl})$

Then, we conducted a one-way repeated measures ANOVA to investigate the effect of stress on time taken to find a target. The results of the test did not show a statistically significant effect of stress on visual search time $(p>0.05)$. Finally, we investigated the effect of stress on the number of errors during visual search tasks. A one-way repeated measures ANOVA did not show an effect of stress on the number of errors.

\subsection{Text Entry Task}

We measured text entry performance in terms of character entry rate, number of total errors, and total error rate as suggested by Soukoreff and MacKenzie [53]. Character entry rate was calculated as the time taken to enter a character, while number of total errors was calculated as the sum of uncorrected and corrected errors. Finally, total error rate was the ratio between the number of total errors and total entered characters. We conducted a one-way repeated measures ANOVA to investigate the effect of stress on time per character entry. The results did not reveal a statistically significant effect of stress on time per character entry $(p=0.056)$, with participants tending to take less time per character entry after post-stress recovery time. Furthermore, a one-way repeated measures ANOVA did not show a statistically significant effect of stress on the number of total errors $(p>0.05)$, nor on total error rate $(p>0.05)$.

As we presented our participants with two types of texts: easy and difficult, we conducted a two-way repeated measures ANOVA, accounting for the effect of difficulty and stress on the number of total errors and total error rate. We did not consider this difference for the character entry rate, as this is already accounted for with text difficulty. The results of the ANOVA showed a statistically significant effect of the difficulty of the text on the number of total errors $(F(1,24)=34.22, p<0.01)$. However, we did not find an interaction effect between 
the difficulty of the text and stress ( $p>0.05$ ). Finally, a two-way repeated measures ANOVA did not show a statistically significant effect of the difficulty of the text on the total error rate $(p>0.01)$.

\subsection{Interviews}

To familiarise ourselves with the data collected during the semi-structured interviews, we read through each response and analysed it using the following process. One researcher printed participant responses and completed initial coding, utilising a data-driven approach based on our aforementioned results. Then, three researchers compared the initial codes and merged these according to their similarity (e.g., "I rushed the task" and "I got progressively slower" merged to "Task completion time"). The three researchers then independently coded participants' responses for each question as based on the initial codebook. We reviewed the final coding to identify similarities that allowed thematic grouping. The main themes are described below.

4.5.1 Psychological Effects of Stress on Perceived Performance. The majority of the participants $(N=19)$ stated that stress affected their behaviour. For instance, participants ended up rushing through the tasks when stressed. Rushing the tasks affected their performance in terms of accuracy during target acquisition tasks. Example quotes are: "I was more annoyed and just wanted to get the tasks done, so I rushed" (P13), "I think because I rushed the task I got less accurate" (P17), "When I'm stressed I get adrenaline, and tap faster, but when I make a mistake I go slower, but still not very accurate" (P14). These findings are in line with our quantitative data, that showed that participants took significantly less time to tap circles when they were stressed as well as they were less accurate.

Participants also reported that stress had an effect on their ability to concentrate and focus. However, the comments regarding concentration were not unanimous. While some participants $(N=8)$ claimed that stress impaired their concentration, others claimed that stress sharpened their focus $(N=5)$. This concords with the fact that, for some participants their perceived performance during visual search and text entry tasks was improved, for example, "I felt I messed up during first few trials as I wasn't paying attention, but stress made me focus better" (P07), "After speech I couldn't concentrate" (P20). While for others, their perceived performance deteriorated: "I forgot the icons as I was stressed" (P04), "I was thinking about the icons, focused on them, but after the arithmetic task I wasn't focused on the icon, and 60\% of the time i just pressed continue and was thinking what was the icon I needed to find" (P17), "After presentation I couldn't focus, when I was doing the icons task I was thinking about my presentation and it took longer time to focus on the icon" (P18).

4.5.2 Physical Effect of Stress on Perceived Performance. We found that stress affected participants not only psychologically, but also physically. Several participants $(N=5)$ claimed that stress had a physical effect on them. All of these participants reported that they felt jittery under stress, and as a result they were less accurate when completing target acquisition tasks. We recorded the following quotes from our participants: "I was more jittery after the speech, my fingers were more shaky" (P09), "My fingers were shivering, especially after the presentation. My head was cloudy, and I was not focused" (P14). These findings are in line with our quantitative results, which show that participants are less accurate in target acquisition task when they are stressed.

4.5.3 Self-Reflection. We also found that several participants reflected on their performance in the speech and arithmetic tasks. This self-reflection was mostly performed during the final part of the study when participants were left alone in the desensitisation room for a post-stress recovery period. While some participants $(N=7)$ expressed their concerns regarding their performance during the arithmetic task: "I was thinking that I could have done better in the arithmetic task", others $(N=7)$ reflected on their performance during the speech task: "I was thinking how bad I did in the presentation" (P16). These comments indicate that even after the stress-inducing tasks during post-stress recovery period, participants were still preoccupied with their performance. 
Table 1. Comparison of the Effects of Situational Impairments against Respective Baseline.

\begin{tabular}{|c|c|c|c|c|c|c|c|c|c|c|c|c|}
\hline & \multicolumn{3}{|c|}{ Baseline } & \multicolumn{9}{|c|}{ Situational Impairments } \\
\hline & Warm & Silent & No stress & Cold & $\begin{array}{c}\text { Music } \\
\text { Fast }\end{array}$ & $\begin{array}{l}\text { Music } \\
\text { Slow }\end{array}$ & $\begin{array}{c}\text { Noise } \\
\text { Indoor }\end{array}$ & $\begin{array}{l}\text { Noise } \\
\text { Outdoor }\end{array}$ & $\begin{array}{c}\text { Speech } \\
\text { Meaningful }\end{array}$ & $\begin{array}{c}\text { Speech } \\
\text { Meaningless }\end{array}$ & Stress & $\begin{array}{c}\text { Post-Stress } \\
\text { Recovery }\end{array}$ \\
\hline $\begin{array}{l}\text { Time to tap } \\
\text { a circle, ms }\end{array}$ & 593.00 & 591.84 & 538.68 & $\begin{array}{l}603.00 \\
+1.7 \%\end{array}$ & $\begin{array}{l}577.76 \\
-2.4 \%\end{array}$ & $\begin{array}{l}574.15 \\
-3.0 \%\end{array}$ & $\begin{array}{l}583.56 \\
-1.4 \%\end{array}$ & $\begin{array}{l}573.56 \\
-3.1 \%\end{array}$ & $\begin{array}{l}592.81 \\
+0.2 \%\end{array}$ & $\begin{array}{l}609.38 \\
+3.0 \%\end{array}$ & $\begin{array}{l}534.77 \\
-0.7 \%\end{array}$ & $\begin{array}{l}520.18 \\
-3.4 \%\end{array}$ \\
\hline $\begin{array}{l}\text { Offset } \\
\text { size, px }\end{array}$ & 41.34 & 39.38 & 46.47 & $\begin{array}{c}42.66 \\
+3.1 \%\end{array}$ & $\begin{array}{r}42.95 \\
+9.1 \%\end{array}$ & $\begin{array}{c}42.11 \\
+6.9 \%\end{array}$ & $\begin{array}{c}41.06 \\
+4.3 \%\end{array}$ & $\begin{array}{c}40.91 \\
+3.9 \%\end{array}$ & $\begin{array}{c}42.90 \\
+8.9 \%\end{array}$ & $\begin{array}{c}39.47 \\
+0.3 \%\end{array}$ & $\begin{array}{c}49.14 \\
+5.7 \%\end{array}$ & $\begin{array}{c}50.20 \\
+8.0 \%\end{array}$ \\
\hline $\begin{array}{l}\text { Time to } \\
\text { memorise } \\
\text { an icon, ms }\end{array}$ & 815.00 & 748.76 & 800.51 & $\begin{array}{l}854.00 \\
+4.8 \%\end{array}$ & $\begin{array}{l}737.71 \\
-1.5 \%\end{array}$ & $\begin{array}{l}745.15 \\
-0.5 \%\end{array}$ & $\begin{array}{l}712.11 \\
-4.9 \%\end{array}$ & $\begin{array}{l}743.56 \\
-0.7 \%\end{array}$ & $\begin{array}{r}753.20 \\
+0.6 \%\end{array}$ & $\begin{array}{l}738.90 \\
-1.3 \%\end{array}$ & $\begin{array}{l}714.52 \\
-4.6 \%\end{array}$ & $\begin{array}{c}702.31 \\
-12.3 \%\end{array}$ \\
\hline $\begin{array}{l}\text { Time to } \\
\text { find an } \\
\text { icon, ms }\end{array}$ & 1632.24 & 1587.74 & 1602.45 & $\begin{array}{l}1942.46 \\
+19.0 \%\end{array}$ & $\begin{array}{c}1564.70 \\
-1.4 \%\end{array}$ & $\begin{array}{r}1753.19 \\
+10.4 \%\end{array}$ & $\begin{array}{c}1543.12 \\
-2.8 \%\end{array}$ & $\begin{array}{c}1633.15 \\
+2.9 \%\end{array}$ & $\begin{array}{c}1637.54 \\
+3.1 \%\end{array}$ & $\begin{array}{c}1520.99 \\
-4.2 \%\end{array}$ & $\begin{array}{c}1496.50 \\
-6.6 \%\end{array}$ & $\begin{array}{c}1506.38 \\
-6.0 \%\end{array}$ \\
\hline $\begin{array}{l}\text { Character } \\
\text { entry rate, } \\
\text { ms/char }\end{array}$ & $\mathrm{n} / \mathrm{a}$ & 454.39 & 560.88 & $\mathrm{n} / \mathrm{a}$ & $\begin{array}{l}488.11 \\
+7.4 \%\end{array}$ & $\begin{array}{r}485.14 \\
+6.8 \%\end{array}$ & $\begin{array}{r}480.96 \\
+5.8 \%\end{array}$ & $\begin{array}{r}523.89 \\
+15.3 \%\end{array}$ & $\begin{array}{c}539.82 \\
+18.8 \%\end{array}$ & $\begin{array}{c}508.99 \\
+12.0 \%\end{array}$ & $\begin{array}{l}547.16 \\
-2.4 \%\end{array}$ & $\begin{array}{c}495.83 \\
-11.6 \%\end{array}$ \\
\hline $\begin{array}{l}\text { Total error } \\
\text { rate, \% }\end{array}$ & $\mathrm{n} / \mathrm{a}$ & 7.54 & 7.46 & $\mathrm{n} / \mathrm{a}$ & $\begin{array}{c}7.45 \\
-1.2 \%\end{array}$ & $\begin{array}{c}7.38 \\
-2.1 \%\end{array}$ & $\begin{array}{c}8.54 \\
+13.3 \%\end{array}$ & $\begin{array}{c}8.22 \\
+9.0 \%\end{array}$ & $\begin{array}{c}7.09 \\
-6.0 \%\end{array}$ & $\begin{array}{c}7.07 \\
-6.2 \%\end{array}$ & $\begin{array}{c}10.50 \\
+40.8 \%\end{array}$ & $\begin{array}{c}9.40 \\
+26.0 \%\end{array}$ \\
\hline
\end{tabular}

Mean values per condition, relative change in $\%$ as compared to baseline. Colour code explanation: Blue - findings of study on ambient temperature [42]. Yellow - findings of study on ambient noise [44]. Gray - findings of this study.

\subsection{Comparison between Situational Impairments}

In this section, we compare stress-, cold- and noise-induced situational impairments. This comparison allows us to increase our understanding on the impact of different situational impairments, thus contributing towards this growing research agenda.

We present the mean values for the following variables in Table 1: time taken to tap a circle, size of the offset, time taken to memorise an icon, time taken to find an icon, character entry rate, and total error rate in typing. The last two variables are not available for the cold-induced situational impairments, as the authors did not quantify performance during the text entry task under cold ambience [42]. Furthermore, we calculate the magnitude of the impact of each situational impairment, when compared to their respective baseline (as a percentage). Given these calculations and the fact that the experimental tasks were identical between the three studies, we are able to directly compare magnitudes of the effects of each factor causing situational impairments (stress, cold ambience [42], ambient noise [44]).

From the table, we observe that when it comes to hitting buttons and icons on the screen (target acquisition) stress has a more detrimental effect than other situational impairments, even than cold. In fact, our results show that during post-stress recovery, participants are less accurate at hitting targets than when they are exposed to cold ambience. Further, stress affected participants differently when compared to noise: character entry rate dropped by $-11.6 \%$ and total error rate increased by $+26.0 \%$.

Interestingly, however, in Table 1 we see although stress is detrimental to target acquisition, its effect is not as acute as the one of music with fast tempo $(+9.1 \%)$, and meaningful speech $(+8.9 \%)$. Nevertheless, we can conclude that the effect of stress is the greatest on memorising time $(-12.3 \%)$ compared to cold or ambient noise. 


\section{DISCUSSION}

Our findings suggest that even though we do not find a significant effect of stress on throughput in the target acquisition task, it does lead participants to favour speed to the detriment of accuracy. We also show that under stress our participants are quicker to memorise an icon during the visual search task. However, our results do not reveal a significant effect of stress on the text entry task. Below we discuss our findings with regard to existing work. We then discuss the benefits and potential future contributions of this research to the HCI community.

\subsection{Effects of Stress on Interaction}

Our results show that the target acquisition time and memorising time are significantly shorter when participants tap circles and complete visual search tasks after the post-stress recovery period. Our findings are in line with prior literature, which shows that people tend to rush and require significantly less time to complete tasks when stressed [33]. Moreover, our participants confirm this behaviour during the interview sessions. They mention that stress caused them anxiety and discomfort, and, hence they rushed through the tasks. Furthermore, the participants report that stress affected their concentration and focus. As a result, they did not pay attention to the icon shown during the visual search task. Instead, they proceeded to the next screen for the sake of completing the task.

Additionally, our results show that stress causes our participants to be less accurate when performing target acquisition tasks. This result also reflects prior literature, which shows that people tend to have higher error rates in completing tasks when stressed [33]. Interestingly, this is also the case in the post-stress recovery measurements. During our interviews the participants claimed that they spent this post-stress recovery period self-reflecting on the tasks, which kept them stressed throughout the recovery period. Consequently, their performance during mobile interaction was negatively affected. Our findings are in line with literature [20], as the authors of the TSST report a high cortisol level in participants during the post-stress recovery period.

Nevertheless, our findings do not show a statistically significant effect of stress on effective throughput in the target acquisition task. This result is in line with prior findings by MacKenzie and Isokoski, where they show that throughput remains constant when target acquisition time and accuracy drop during tapping tasks [28]. This can be explained by the fact that throughput accounts for both speed and accuracy and as these two variables change in opposite directions, throughput does not significantly change [28]. However, we note that while throughput remained relatively constant in this scenario, this is likely not to be the case when a stressed user interacts with a real-world application that has a cost associated with errors.

Furthermore, our results show that text difficulty had a larger effect on performance during the text entry task than participant stress levels. Regarding the text entry task several participants $(N=9)$ report that they made comparatively more errors after they experienced stress. While our quantitative results show a tendency towards this being the case, this effect was not statistically significant.

\subsection{Contrasting Situational Impairments}

Previous work has highlighted the importance of accumulating knowledge within different areas of $\mathrm{HCI}$ for the formation of paramount research themes [24, 25]. Here, we contribute towards this notion by contrasting the magnitudes of the effects of different situational impairments on mobile interaction.

Previous work, utilising the same tasks reported in this study, has shown that text entry rate is significantly affected by ambient noise, particularly when considering meaningful speech (speech in a language the participant understands) [44]. However, in our study, participants' performance during text entry (in terms of character entry rate) was not significantly affected by stress, suggesting that stress has a smaller effect on participants' ability to perform text entry compared to meaningful speech. This is not surprising given the nature of the text entry task. Meaningful speech is particularly disruptive when a person is thinking about what they should say or write [30]. 
Furthermore, in Table 1 we can see that the effect of cold ambience on the target access time was opposite to the one of stress. To be precise, target acquisition time was longer in cold environment [42]; however, it was shorter during the post-stress recovery period (as compared to the relative baselines). This can be explained by the nature of the environment, as cold ambience decreases manual dexterity [60], and hence, increases the completion time for the tasks involving fine-motor manipulations [66]. Whereas, stress is known to increase anxiety [23], thus leading people to rush to complete the tasks [33]. This effect was also highlighted in our qualitative data; and is applicable not only for tasks requiring fine-motor skills, but also for visual search tasks. For example, our participants spent less time on memorising and searching for icons, as compared to the participants from the study presented in [42], where they took longer time both to memorise and find icons. Interestingly, cold-, noise-, and stress-induced situational impairments have a roughly comparable adverse effect on the tapping accuracy. This can be explained by the nature of the task, as both external and internal factors affect accuracy in only one direction - negative. The only difference is the magnitude of the effect, shown in detail in Table 1.

\subsection{Implications for Research and Design}

Previous research has emphasised the importance of understanding the effects of situational impairments on mobile interaction, as it further enables the construction of sensing mechanisms to detect situational impairments and, thus, adapt the interface accordingly [43]. Our study shows that stress can impair mobile interaction, and, hence, this information can be used to adapt the interface to accommodate such impairments. For instance, to mitigate the effects of stress-induced situational impairments on target acquisition tasks, it is possible to increase target sizes or use techniques proposed in the literature to improve input accuracy, such as GraspZoom [34] and Fat Thumb [6]. GraspZoom allows zooming on a particular part of the screen with a long press using only the thumb [34]. Fat Thumb includes only two interaction gesture modes: panning and zooming, with gesture being defined by the contact size of the thumb [6]. We acknowledge that the effect size of stress on interaction performance might not seem to be strong enough to impair the user experience in real world scenario. However, we expect that when completing more complex tasks on a smartphone, the effect size would be more pronounced.

However, it is necessary to first detect stress before adaptation actually takes place. Wearable sensors have successfully been leveraged for this purpose by measuring physiological measurements such as skin conductivity [50] and heart rate variability [56]. In our study we use HRV measured through a wearable device alongside self-reports, to confirm that participants are indeed being affected by the TSST protocol. Beyond sensor data and self-reports, previous work has shown that daily stress can also be inferred from smartphone usage, personality traits, and weather data [5]. Moreover, the Intel Mobile Heart Health [36] prototype uses data from sources such as mobile phone-based ecological momentary assessment and a small electrocardiograph sensor with an accelerometer to detect changes in heart rate variability, activity, and mood. If individualised threshold values are reached, the mobile phone delivers cognitive behavioural and mindfulness techniques designed to reduce stress [36]. Within the literature on situational impairments, there are also several examples of sensor information being used to detect certain contextual factors. For example, Goel et al. [11] used a smartphone's accelerometer to detect if the user is walking. Similarly, Sarsenbayeva et al. suggested using the smartphone's battery temperature to detect cold-induced situational impairments [45].

Ultimately, this detection should ideally be performed unobtrusively and without creating additional stress [14]. Unobtrusive and continuous stress detection would benefit mobile device users. For example, an individual being aware of their exposure to stress, could change their behaviour to eliminate unnecessary stressors [14]. A smartphone's operating system that understands the user's application usage behaviour $[17,58]$, and having detected the user being stressed, could prevent unnecessary notifications or updates being presented [14], and adapt the interface to minimise errors. 


\subsection{Limitations}

We acknowledge a number of limitations in our study. First, the study settings were strictly controlled. It is possible that in a more naturalistic environment, participants can experience a stronger level of stress. However, it was necessary to follow an established protocol to strictly control stress induction and avoid causing harm to our participants. The types of smartphone tasks presented in this study were limited to target acquisition, visual search, and text entry, whereas, in a naturalistic environment, users may perform more complex tasks, requiring more cognitive demand [2]. Nevertheless, in this study we reported a statistically significant effect of stress on basic smartphone tasks (e.g., target acquisition and visual search), which suggests that this effect would be more pronounced when completing more complex tasks during mobile interaction. Furthermore, we did not find any statistically significant effect of stress on text entry tasks. However, we acknowledge that stress might have an impact on text entry performance with more complex sentences. Future research is needed to investigate this assumption. Finally, we restricted our participants to only one interaction mode with the smartphone - using the index finger. We argue that controlling the interaction mode was necessary to draw a fair comparison between the effects of stress, ambient noise [44], and cold ambience [42].

\section{CONCLUSION}

In this work, we investigate the effects of stress on performance on three common mobile interaction tasks: target acquisition, visual search, and text entry. We use the Trier Social Stress Test to induce stress on participants. Our findings show that the target acquisition time and the time to memorise an icon become significantly shorter during stress and post-stress recovery periods. We also show that stress deteriorates participants' accuracy during target acquisition tasks. These findings can be used to inform how interfaces should adapt to accommodate such impairments.

We then compare the effects of cold-, noise-, and stress-induced situational impairments on performance during mobile interaction. Our results show that the effect was more pronounced on target acquisition tasks in terms of task completion time and accuracy. Our findings on the effects of stress on mobile interaction extend our understanding of situational impairments. This knowledge is paramount to inform the development of adaptive interfaces that accommodate such impairments.

\section{ACKNOWLEDGMENTS}

This work is partially funded by a Samsung Global Research Outreach grant and JST ERATO Grant Number JPMJER1501, Japan. Eduardo Velloso is the recipient of an Australian Research Council Discovery Early Career Award (Project Number: DE180100315) funded by the Australian Government.

\section{REFERENCES}

[1] 2007. Work-related stress. http://www.eurofound.europa.eu/ewco/reports/TN0502TR01/TN0502TR01.pdf

[2] Brian P Bailey, Joseph A Konstan, and John V Carlis. 2001. The Effects of Interruptions on Task Performance, Annoyance, and Anxiety in the User Interface.. In Interact, Vol. 1. 593-601.

[3] Gerald Bauer and Paul Lukowicz. 2012. Can smartphones detect stress-related changes in the behaviour of individuals?. In Pervasive Computing and Communications Workshops (PERCOM Workshops), 2012 IEEE International Conference on. IEEE, 423-426.

[4] Gary G Berntson and John T Cacioppo. 2004. Heart rate variability: Stress and psychiatric conditions. Dynamic electrocardiography (2004), 57-64.

[5] Andrey Bogomolov, Bruno Lepri, Michela Ferron, Fabio Pianesi, and Alex Sandy Pentland. 2014. Daily stress recognition from mobile phone data, weather conditions and individual traits. In Proceedings of the 22nd ACM international conference on Multimedia. ACM, $477-486$.

[6] Sebastian Boring, David Ledo, Xiang’Anthony' Chen, Nicolai Marquardt, Anthony Tang, and Saul Greenberg. 2012. The fat thumb: using the thumb's contact size for single-handed mobile interaction. In Proceedings of the 14th international conference on Human-computer interaction with mobile devices and services. ACM, 39-48. 
[7] Wolfram Boucsein. 2012. Electrodermal activity. Springer Science \& Business Media.

[8] Jason J Braithwaite, Derrick G Watson, Robert Jones, and Mickey Rowe. 2013. A guide for analysing electrodermal activity (EDA) \& skin conductance responses (SCRs) for psychological experiments. Psychophysiology 49, 1 (2013), 1017-1034.

[9] Sheldon Cohen, Tom Kamarck, and Robin Mermelstein. 1983. A global measure of perceived stress. fournal of health and social behavior (1983), 385-396.

[10] Marc Exposito, Rosalind W Picard, and Javier Hernandez. 2018. Affective keys: towards unobtrusive stress sensing of smartphone users. In Proceedings of the 20th International Conference on Human-Computer Interaction with Mobile Devices and Services Adjunct. ACM, 139-145.

[11] Mayank Goel, Leah Findlater, and Jacob Wobbrock. 2012. WalkType: Using Accelerometer Data to Accomodate Situational Impairments in Mobile Touch Screen Text Entry. In Proceedings of the SIGCHI Conference on Human Factors in Computing Systems (CHI '12). ACM, New York, NY, USA, 2687-2696. https://doi.org/10.1145/2207676.2208662

[12] Jorge Goncalves, Zhanna Sarsenbayeva, Niels van Berkel, Chu Luo, Simo Hosio, Sirkka Risanen, Hannu Rintamäki, and Vassilis Kostakos. 2017. Tapping Task Performance on Smartphones in Cold Temperature. Interacting with Computers 29, 3 (2017), 355-367. https://doi.org/10.1093/iwc/iww029

[13] Niels Henze, Enrico Rukzio, and Susanne Boll. 2011. 100,000,000 Taps: Analysis and Improvement of Touch Performance in the Large. In Proceedings of the 13th International Conference on Human Computer Interaction with Mobile Devices and Services (MobileHCI '11). ACM, New York, NY, USA, 133-142. https://doi.org/10.1145/2037373.2037395

[14] Javier Hernandez, Pablo Paredes, Asta Roseway, and Mary Czerwinski. 2014. Under pressure: sensing stress of computer users. In Proceedings of the SIGCHI conference on Human factors in computing systems. ACM, 51-60.

[15] Nis Hjortskov, Dag Rissén, Anne Katrine Blangsted, Nils Fallentin, Ulf Lundberg, and Karen Søgaard. 2004. The effect of mental stress on heart rate variability and blood pressure during computer work. European journal of applied physiology 92, 1-2 (2004), 84-89.

[16] Marko Jelici, Elke Geraerts, Harald Merckelbach, and Ramona Guerrieri. 2004. Acute stress enhances memory for emotional words, but impairs memory for neutral words. International fournal of Neuroscience 114, 10 (2004), 1343-1351.

[17] Simon L. Jones, Denzil Ferreira, Simo Hosio, Jorge Goncalves, and Vassilis Kostakos. 2015. Revisitation Analysis of Smartphone App Use. In Proceedings of the 2015 ACM International foint Conference on Pervasive and Ubiquitous Computing (UbiComp '15). ACM, New York, NY, USA, 1197-1208. https://doi.org/10.1145/2750858.2807542

[18] Indika Karunaratne, Ajantha Sanjeewa Atukorale, and Hemamali Perera. 2011. Surveillance of human-computer interactions: A way forward to detection of users' Psychological Distress. In Humanities, Science and Engineering (CHUSER), 2011 IEEE Colloquium on. IEEE, 491-496.

[19] J Peter Kincaid, Robert P Fishburne Jr, Richard L Rogers, and Brad S Chissom. 1975. Derivation of new readability formulas (automated readability index, fog count and flesch reading ease formula) for navy enlisted personnel. Technical Report. Naval Technical Training Command Millington TN Research Branch.

[20] Clemens Kirschbaum, Karl-Martin Pirke, and Dirk H Hellhammer. 1993. The 'Trier Social Stress Test'-a tool for investigating psychobiological stress responses in a laboratory setting. Neuropsychobiology 28, 1-2 (1993), 76-81.

[21] Clemens Kirschbaum, Oliver T Wolf, Mark May, W Wippich, and Dirk H Hellhammer. 1996. Stress-and treatment-induced elevations of cortisol levels associated with impaired declarative memory in healthy adults. Life sciences 58, 17 (1996), 1475-1483.

[22] Sabrina Kuhlmann, Marcel Piel, and Oliver T Wolf. 2005. Impaired memory retrieval after psychosocial stress in healthy young men. Journal of Neuroscience 25, 11 (2005), 2977-2982.

[23] Richard S Lazarus, James Deese, and Sonia F Osler. 1952. The effects of psychological stress upon performance. Psychological bulletin 49, 4 (1952), 293.

[24] Yong Liu, Jorge Goncalves, Denzil Ferreira, Simo Hosio, and Vassilis Kostakos. 2014. Identity Crisis of Ubicomp?: Mapping 15 Years of the Field's Development and Paradigm Change. In Proceedings of the 2014 ACM International foint Conference on Pervasive and Ubiquitous Computing (UbiComp '14). ACM, New York, NY, USA, 75-86. https://doi.org/10.1145/2632048.2632086

[25] Yong Liu, Jorge Goncalves, Denzil Ferreira, Bei Xiao, Simo Hosio, and Vassilis Kostakos. 2014. CHI 1994-2013: Mapping Two Decades of Intellectual Progress Through Co-word Analysis. In Proceedings of the 32nd Annual ACM Conference on Human Factors in Computing Systems (CHI '14). ACM, New York, NY, USA, 3553-3562.

[26] SJ Lupien, S Gaudreau, BM Tchiteya, F Maheu, S Sharma, NPV Nair, RL Hauger, BS McEwen, and MJ Meaney. 1997. Stress-induced declarative memory impairment in healthy elderly subjects: relationship to cortisol reactivity. The fournal of Clinical Endocrinology \& Metabolism 82, 7 (1997), 2070-2075.

[27] Sonia J Lupien, Christian J Gillin, and Richard L Hauger. 1999. Working memory is more sensitive than declarative memory to the acute effects of corticosteroids: A dose-response study in humans. Behavioral neuroscience 113, 3 (1999), 420.

[28] I Scott MacKenzie and Poika Isokoski. 2008. Fitts' throughput and the speed-accuracy tradeoff. In Proceedings of the SIGCHI Conference on Human Factors in Computing Systems. ACM, 1633-1636.

[29] Dorothy Irene Marquart. 1948. The pattern of punishment and its relation to abnormal fixation in adult human subjects. The fournal of general psychology 39, 1 (1948), 107-144.

Proc. ACM Interact. Mob. Wearable Ubiquitous Technol., Vol. 3, No. 1, Article 24. Publication date: March 2019. 
[30] Randi C Martin, Michael S Wogalter, and Janice G Forlano. 1988. Reading comprehension in the presence of unattended speech and music. Journal of memory and language 27, 4 (1988), 382-398.

[31] Daniel J McDuff, Javier Hernandez, Sarah Gontarek, and Rosalind W Picard. 2016. Cogcam: Contact-free measurement of cognitive stress during computer tasks with a digital camera. In Proceedings of the 2016 CHI Conference on Human Factors in Computing Systems. ACM, 4000-4004.

[32] Fred McKinney. 1933. Certain emotional factors in learning and efficiency. The fournal of General Psychology 9, 1 (1933), $101-116$.

[33] Fred McKinney, George B Strother, Ruth R Hines, and Ruth A Allee. 1951. Experimental frustration in a group test situation. The fournal of Abnormal and Social Psychology 46, 3 (1951), 316.

[34] Takashi Miyaki and Jun Rekimoto. 2009. GraspZoom: zooming and scrolling control model for single-handed mobile interaction. In Proceedings of the 11th International Conference on Human-Computer Interaction with Mobile Devices and Services. ACM, 11.

[35] Sachi Mizobuchi, Mark Chignell, and David Newton. 2005. Mobile text entry: relationship between walking speed and text input task difficulty. In Proceedings of the 7th international conference on Human computer interaction with mobile devices \& services. ACM, 122-128.

[36] Margaret Morris and Farzin Guilak. 2009. Mobile heart health: project highlight. IEEE Pervasive Computing 2 (2009), 57-61.

[37] John W Newcomer, Gregg Selke, Angela K Melson, Tamara Hershey, Suzanne Craft, Katherine Richards, and Amy L Alderson. 1999. Decreased memory performance in healthy humans induced by stress-level cortisol treatment. Archives of general psychiatry 56, 6 (1999), 527-533.

[38] Alexander Ng, Stephen A. Brewster, and John H. Williamson. 2014. Investigating the Effects of Encumbrance on One- and Two- Handed Interactions with Mobile Devices. In Proceedings of the SIGCHI Conference on Human Factors in Computing Systems (CHI '14). ACM, New York, NY, USA, 1981-1990. https://doi.org/10.1145/2556288.2557312

[39] Jessica D Payne, Lynn Nadel, John JB Allen, Kevin GF Thomas, and W Jake Jacobs. 2002. The effects of experimentally induced stress on false recognition. Memory 10, 1 (2002), 1-6.

[40] Manuel Rodrigues, Sérgio Gonçalves, Davide Carneiro, Paulo Novais, and Florentino Fdez-Riverola. 2013. Keystrokes and clicks: Measuring stress on e-learning students. In Management Intelligent Systems. Springer, 119-126.

[41] Akane Sano and Rosalind W Picard. 2013. Stress recognition using wearable sensors and mobile phones. In Affective Computing and Intelligent Interaction (ACII), 2013 Humaine Association Conference on. IEEE, 671-676.

[42] Zhanna Sarsenbayeva, Jorge Goncalves, Juan García, Simon Klakegg, Sirkka Rissanen, Hannu Rintamäki, Jari Hannu, and Vassilis Kostakos. 2016. Situational Impairments to Mobile Interaction in Cold Environments. In Proceedings of the 2016 ACM International foint Conference on Pervasive and Ubiquitous Computing (UbiComp '16). ACM, New York, NY, USA, 85-96. https://doi.org/10.1145/2971648.2971734

[43] Zhanna Sarsenbayeva, Niels van Berkel, Chu Luo, Vassilis Kostakos, and Jorge Goncalves. 2017. Challenges of situational impairments during interaction with mobile devices. In Proceedings of the 29th Australian Conference on Computer-Human Interaction. ACM, 477-481.

[44] Zhanna Sarsenbayeva, Niels van Berkel, Eduardo Velloso, Vassilis Kostakos, and Jorge Goncalves. 2018. Effect of Distinct Ambient Noise Types on Mobile Interaction. Proceedings of the ACM on Interactive, Mobile, Wearable and Ubiquitous Technologies 2, 3 (2018).

[45] Zhanna Sarsenbayeva, Niels van Berkel, Aku Visuri, Sirkka Rissanen, Hannu Rintamäki, Vassilis Kostakos, and Jorge Goncalves. 2017. Sensing Cold-Induced Situational Impairments in Mobile Interaction Using Battery Temperature. Proc. ACM Interact. Mob. Wearable Ubiquitous Technol. 1, 3, Article 98 (Sept. 2017), 9 pages. https://doi.org/10.1145/3130963

[46] Sidas Saulynas, Lawrence Burgee, and Ravi Kuber. 2017. All situational impairments are not created equal: A classification system for situational impairment events and the unique nature of severely constraining situational impairments. iConference 2017 (2017).

[47] Michael A Sayette. 1999. Does drinking reduce stress? Alcohol research and health 23, 4 (1999), 250-255.

[48] Bastian Schildbach and Enrico Rukzio. 2010. Investigating selection and reading performance on a mobile phone while walking. In Proceedings of the 12th international conference on Human computer interaction with mobile devices and services. ACM, 93-102.

[49] Andrew Sears, Min Lin, Julie Jacko, and Yan Xiao. 2003. When computers fade: Pervasive computing and situationally-induced impairments and disabilities. In HCI International, Vol. 2. 1298-1302.

[50] Cornelia Setz, Bert Arnrich, Johannes Schumm, Roberto La Marca, Gerhard Tröster, and Ulrike Ehlert. 2010. Discriminating stress from cognitive load using a wearable EDA device. IEEE Transactions on information technology in biomedicine 14, 2 (2010), 410-417.

[51] Riccardo Sioni and Luca Chittaro. 2015. Stress detection using physiological sensors. Computer 48, 10 (2015), 26-33.

[52] Aaron Smith. 1967. The serial sevens subtraction test. Archives of Neurology 17, 1 (1967), 78-80.

[53] R William Soukoreff and I Scott MacKenzie. 2003. Metrics for text entry research: an evaluation of MSD and KSPC, and a new unified error metric. In Proceedings of the SIGCHI conference on Human factors in computing systems. ACM, 113-120.

[54] Charles D Spielberger. 2010. State-Trait anxiety inventory. Wiley Online Library.

[55] Mark A Staal. 2004. Stress, cognition, and human performance: A literature review and conceptual framework. (2004).

[56] David Sun, Pablo Paredes, and John Canny. 2014. MouStress: detecting stress from mouse motion. In Proceedings of the SIGCHI conference on Human factors in computing systems. ACM, 61-70.

[57] Garreth W. Tigwell, David R. Flatla, and Rachel Menzies. 2018. It's Not Just the Light: Understanding the Factors Causing Situational Visual Impairments During Mobile Interaction. In Proceedings of the 10th Nordic Conference on Human-Computer Interaction (NordiCHI '18). ACM, New York, NY, USA. https://doi.org/10.1145/3240167.3240207 
[58] Niels van Berkel, Chu Luo, Theodoros Anagnostopoulos, Denzil Ferreira, Jorge Goncalves, Simo Hosio, and Vassilis Kostakos. 2016. A Systematic Assessment of Smartphone Usage Gaps. In Proceedings of the 2016 CHI Conference on Human Factors in Computing Systems (CHI '16). ACM, New York, NY, USA, 4711-4721. https://doi.org/10.1145/2858036.2858348

[59] Ruud Van den Bos, Marlies Harteveld, and Hein Stoop. 2009. Stress and decision-making in humans: performance is related to cortisol reactivity, albeit differently in men and women. Psychoneuroendocrinology 34, 10 (2009), 1449-1458.

[60] JA Vaughan, EA Higgins, GE Funkhouser, Elinore M Galerston, et al. 1968. Effects of body thermal state on manual performance. Aerospace medicine 39, 12 (1968), 1310-1315.

[61] Elinor Verville. 1946. The effect of emotional and motivational sets on the perception of incomplete pictures. The Pedagogical Seminary and Journal of Genetic Psychology 69, 2 (1946), 133-145.

[62] J Wahlström, M Hagberg, P Johnson, J Svensson, and D Rempel. 2002. Influence of time pressure and verbal provocation on physiological and psychological reactions during work with a computer mouse. European journal of applied physiology 87, 3 (2002), 257-263.

[63] Jacob O Wobbrock. 2006. The future of mobile device research in HCI. In CHI 2006 workshop proceedings: what is the next generation of human-computer interaction. 131-134.

[64] Owen M Wolkowitz, Victor I Reus, Herbert Weingartner, Karen Thompson, Alan Breier, et al. 1990. Cognitive effects of corticosteroids. The American journal of psychiatry 147, 10 (1990), 1297.

[65] Robert M Yerkes and John D Dodson. 1908. The relation of strength of stimulus to rapidity of habit-formation. fournal of comparative neurology and psychology 18, 5 (1908), 459-482.

[66] Mark A Yeshnik. 1988. The variability of German winter temperature in relation to human performance and its implications for tactical military operations. Technical Report. Army Military Personnel Center Alexandria VA.

[67] AH Young, BJ Sahakian, TW Robbins, and PJ Cowen. 1999. The effects of chronic administration of hydrocortisone on cognitive function in normal male volunteers. Psychopharmacology 145, 3 (1999), 260-266.

Received November 2018; revised January 2019; accepted January 2019

Proc. ACM Interact. Mob. Wearable Ubiquitous Technol., Vol. 3, No. 1, Article 24. Publication date: March 2019. 